\title{
The Wright functions as solutions of the time-fractional diffusion equation
}

\author{
Francesco Mainardi ${ }^{\mathrm{a}, *}$, Gianni Pagnini ${ }^{\mathrm{b}}$ \\ a Dipartimento di Fisica, Università di Bologna and INFN, Sezione di Bologna, Via Irnerio 46, \\ I-40126 Bologna, Italy \\ b Istituto per le Scienze dell'Atmosfera e del Clima del CNR, Via Gobetti 101, I-40129 Bologna, Italy
}

\begin{abstract}
We revisit the Cauchy problem for the time-fractional diffusion equation, which is obtained from the standard diffusion equation by replacing the first-order time derivative with a fractional derivative of order $\beta \in(0,2]$. By using the Fourier-Laplace transforms the fundamentals solutions (Green functions) are shown to be high transcendental functions of the Wright-type that can be interpreted as spatial probability density functions evolving in time with similarity properties. We provide a general representation of these functions in terms of Mellin-Barnes integrals useful for numerical computation.
\end{abstract}

(C) 2002 Elsevier Science Inc. All rights reserved.

Keywords: Fractional derivatives; Laplace transforms; Fourier transforms; Mellin-Barnes integrals; Mittag-Leffler functions; Wright functions; Fox $H$-functions

\section{Introduction}

Time-fractional diffusion equations, obtained from the standard diffusion equation by replacing the first-order time derivative by a fractional derivative (of order $0<\beta \leqslant 2$, in Riemann-Liouville or Caputo sense), have been treated in different contexts by a number of authors, see, e.g. the reviews in $[1,13,19]$, and references therein. In this paper we intend to provide more insights for the

\footnotetext{
* Corresponding author.

E-mail address: mainardi@bo.infn.it (F. Mainardi).
} 
fundamental solutions of the general time-fractional diffusion equation, based on the recent results by Mainardi et al. [15].

By time-fractional diffusion equation we mean the evolution equation

$$
\frac{\partial^{\beta}}{\partial t^{\beta}} u(x, t)=\frac{\partial^{2}}{\partial x^{2}} u(x, t), \quad 0<\beta \leqslant 2, x \in \mathbb{R}, \quad t \in \mathbb{R}_{0}^{+},
$$

where the time-fractional derivative is intended in the Caputo sense. For a detailed discussion on this fractional derivative we refer the reader to e.g. $[8,20]$. When $\beta$ is not integer $(\beta \neq 1,2)$ the L.H.S. of $(1.1)$ is intended to be

$$
\frac{\partial^{\beta}}{\partial t^{\beta}} u(x, t):= \begin{cases}\frac{1}{\Gamma(1-\beta)} \int_{0}^{t}\left[\frac{\partial}{\partial \tau} u(x, \tau)\right] \frac{\mathrm{d} \tau}{(t-\tau)^{\beta}}, & \text { if } 0<\beta<1, \\ \frac{1}{\Gamma(2-\beta)} \int_{0}^{t}\left[\frac{\partial^{2}}{\partial \tau^{2}} u(x, \tau)\right] \frac{\mathrm{d} \tau}{(t-\tau)^{\beta-1}}, & \text { if } 1<\beta<2 .\end{cases}
$$

When $\beta$ is integer $(\beta=1,2)$ the R.H.S. of (1.2) is intended to reduce to the corresponding partial derivative of integer order, namely we recover, for $\beta=1$, the diffusion equation:

$$
\frac{\partial}{\partial t} u(x, t)=\frac{\partial^{2}}{\partial x^{2}} u(x, t), \quad x \in \mathbb{R}, \quad t \in \mathbb{R}_{0}^{+},
$$

for $\beta=2$, the D'Alembert wave equation:

$$
\frac{\partial^{2}}{\partial t^{2}} u(x, t)=\frac{\partial^{2}}{\partial x^{2}} u(x, t), \quad x \in \mathbb{R}, \quad t \in \mathbb{R}_{0}^{+} .
$$

For $1<\beta<2$ the fractional equation in (1.1) is expected to interpolate (1.3) and (1.4), thus in this case it could be referred to as the time-fractional diffusionwave equation. Suitable integrations allow us to eliminate the time-fractional derivative in (1.1) and obtain the integro-differential equations:

if $0<\beta \leqslant 1$,

$$
u(x, t)=u\left(x, 0^{+}\right)+\frac{1}{\Gamma(\beta)} \int_{0}^{t}\left[\frac{\partial^{2}}{\partial x^{2}} u(x, \tau)\right](t-\tau)^{\beta-1} \mathrm{~d} \tau,
$$

if $1<\beta \leqslant 2$,

$$
u(x, t)=u\left(x, 0^{+}\right)+t u_{t}\left(x, 0^{+}\right)+\frac{1}{\Gamma(\beta)} \int_{0}^{t}\left[\frac{\partial^{2}}{\partial x^{2}} u(x, \tau)\right](t-\tau)^{\beta-1} \mathrm{~d} \tau .
$$

In order to correctly formulate and solve the Cauchy problem for (1.1) we have to select explicit initial conditions concerning $u\left(x, 0^{+}\right)$if $0<\beta \leqslant 1$ and $u\left(x, 0^{+}\right), u_{t}\left(x, 0^{+}\right)$if $1<\beta \leqslant 2$. If $\phi(x)$ and $\psi(x)$ denote sufficiently well-behaved 
real functions defined on $\mathbb{R}$, the Cauchy problem consists in finding the solution of (1.1) subjected to the initial conditions:

$$
\begin{aligned}
& u\left(x, 0^{+}\right)=\phi(x), \quad x \in \mathbb{R}, \text { if } 0<\beta \leqslant 1, \\
& u\left(x, 0^{+}\right)=\phi(x), \quad u_{t}\left(x, 0^{+}\right)=\psi(x), \quad x \in \mathbb{R}, \text { if } 1<\beta \leqslant 2 .
\end{aligned}
$$

We note that if we set $\psi(x) \equiv 0$ in $(1.7 \mathrm{~b})$ we ensure the continuous dependence of the corresponding solution with respect to the parameter $\beta$ in the transition from $\beta=1^{-}$to $\beta=1^{+}$as it turns out by comparing Eqs. (1.5) and (1.6).

\section{The Green functions: scaling and similarity properties}

The Cauchy problems can be conveniently treated by making use of the most common integral transforms, i.e. the Fourier transform (in space) and the Laplace transform (in time). ${ }^{1}$ Indeed, the composite Fourier-Laplace transforms of the solutions of the two Cauchy problems:

(a) $\{(1.1)+(1.7 \mathrm{a})\}$ if $0<\beta \leqslant 1$,

(b) $\{(1.1)+(1.7 b)\}$ if $1<\beta \leqslant 2$,

turn out to satisfy the following algebraic equations

$$
\begin{aligned}
& -\kappa^{2} \widehat{\widetilde{\boldsymbol{u}}}(\kappa, s)=s^{\beta} \widehat{\widetilde{\boldsymbol{u}}}(\kappa, s)-s^{\beta-1} \widehat{\phi}(\kappa), \quad 0<\beta \leqslant 1, \\
& -\kappa^{2} \widehat{\widetilde{\boldsymbol{u}}}(\kappa, s)=s^{\beta} \widehat{\widetilde{\boldsymbol{u}}}(\kappa, s)-s^{\beta-1} \widehat{\boldsymbol{\phi}}(\kappa)-s^{\beta-2} \widehat{\psi}(\kappa), \quad 1<\beta \leqslant 2,
\end{aligned}
$$

from which we obtain

$$
\widehat{\widetilde{u}}(\kappa, s)=\frac{s^{\beta-1}}{s^{\beta}+\kappa^{2}} \widehat{\phi}(\kappa), \quad 0<\beta \leqslant 1,
$$

\footnotetext{
${ }^{1}$ In what follows we shall meet only functions that are defined and continuous in $x \in \mathbb{R}$ and/or $t \in(0, T), \forall T>0$ except, possibly, at isolated points where these functions can be infinite. Following Marichev [17] we restrict our attention to the classes of such functions for which the Riemann improper integrals in $x$ and in $t$ absolutely converges on $\mathbb{R}$ and $(0, T), \forall T>0$, respectively. We denote these classes as $L^{\mathrm{c}}(\mathbb{R}), L^{\mathrm{c}}(0, T)$. Let

$$
\widehat{f}(\kappa)=\mathscr{F}\{f(x) ; \kappa\}=\int_{-\infty}^{+\infty} \mathrm{e}^{+\mathrm{i} \kappa x} f(x) \mathrm{d} x, \quad \kappa \in \mathbb{R}
$$

denote the Fourier transform of a function $f(x) \in L^{\mathrm{c}}(\mathbb{R})$, and let

$$
\widetilde{f}(s)=\mathscr{L}\{f(t) ; s\}=\int_{0}^{\infty} \mathrm{e}^{-s t} f(t) \mathrm{d} t, \quad \mathfrak{R}(s)>a_{f},
$$

denote the Laplace transform of a function $f(t) \in L^{\mathrm{c}}(0, T)$. We denote by $\stackrel{\mathscr{F}}{\leftrightarrow}$ and $\stackrel{\mathscr{L}}{\leftrightarrow}$ the juxtaposition of a function with its Fourier and Laplace transform, respectively.
} 


$$
\widehat{\widetilde{u}}(\kappa, s)=\frac{s^{\beta-1}}{s^{\beta}+\kappa^{2}} \widehat{\phi}(\kappa)+\frac{s^{\beta-2}}{s^{\beta}+\kappa^{2}} \widehat{\psi}(\kappa), \quad 1<\beta \leqslant 2 .
$$

By fundamental solutions (or Green functions) of the above Cauchy problems we mean the (generalized) solutions corresponding to the initial conditions

$$
\begin{aligned}
& G_{\beta}^{(1)}\left(x, 0^{+}\right)=\delta(x), \quad \text { if } 0<\beta \leqslant 1, \\
& \left\{\begin{array} { l } 
{ G _ { \beta } ^ { ( 1 ) } ( x , 0 ^ { + } ) = \delta ( x ) , } \\
{ \frac { \partial } { \partial t } G _ { \beta } ^ { ( 1 ) } ( x , 0 ^ { + } ) = 0 , }
\end{array} \quad \left\{\begin{array}{l}
G_{\beta}^{(2)}\left(x, 0^{+}\right)=0, \\
\frac{\partial}{\partial t} G_{\beta}^{(2)}\left(x, 0^{+}\right)=\delta(x),
\end{array} \quad \text { if } 1<\beta \leqslant 2 .\right.\right.
\end{aligned}
$$

Here $\delta(x)$ is the delta-Dirac generalized function whose (generalized) Fourier transform is known to be one. Thus, the Fourier-Laplace transforms of these Green functions turn out to be

$$
\widehat{\widetilde{G_{\beta}^{(j)}}}(\kappa, s)=\frac{s^{\beta-j}}{s^{\beta}+\kappa^{2}}, \quad 0<\beta \leqslant 2, \quad j=1,2 .
$$

We note that the function $G_{\beta}^{(2)}(x, t)$ along with its Fourier-Laplace transform is well defined also for $0<\beta \leqslant 1$ even if it loses its meaning of being a fundamental solution of (1.1). Then, by recalling the Fourier convolution property in the inversion of the Fourier-Laplace transforms of (2.2a) and (2.2b), we note that the Green functions allow us the represent the solutions of the above two Cauchy problems through the relevant integral formulas

$$
\begin{aligned}
& u(x, t)=\int_{-\infty}^{+\infty} G_{\beta}^{(1)}(\xi, t) \phi(x-\xi) \mathrm{d} \xi, \quad 0<\beta \leqslant 1, \\
& u(x, t)=\int_{-\infty}^{+\infty}\left[G_{\beta}^{(1)}(\xi, t) \phi(x-\xi)+G_{\beta}^{(2)}(\xi, t) \psi(x-\xi)\right] \mathrm{d} \xi, \quad 1<\beta \leqslant 2 .
\end{aligned}
$$

By using the known scaling rules for the Fourier and Laplace transforms, and introducing the similarity variable $x / t^{\beta / 2}$, we infer from (2.4) (thus without inverting the two transforms) the scaling properties of the Green functions,

$$
G_{\beta}^{(1)}(x, t)=t^{-\beta / 2} K_{\beta}^{(1)}\left(x / t^{\beta / 2}\right), \quad G_{\beta}^{(2)}(x, t)=t^{-\beta / 2+1} K_{\beta}^{(2)}\left(x / t^{\beta / 2}\right),
$$

where the one-variable functions $K_{\beta}^{(1)}(x), K_{\beta}^{(2)}(x)$ are referred to as the reduced Green functions. We note that all Green functions are symmetric with respect to $x$ and

$$
K_{\beta}^{(j)}(x)=G_{\beta}^{(j)}(x, 1)=K_{\beta}^{(j)}(-x), \quad j=1,2 .
$$




\section{Mellin-Barnes integral representation of the Green functions}

To determine the two Green functions in the space-time domain we can follow two alternative strategies related to the different order in carrying out the inversion of the Fourier-Laplace transforms in (2.4), (2.5a) and (2.5b). Indeed we can

(S1): invert the Fourier transforms getting $\widetilde{G_{\beta}^{(1)}}(x, s), \widetilde{G_{\beta}^{(2)}}(x, s)$, and then invert

these Laplace transforms,
(S2): invert the Laplace transforms getting $\widehat{G_{\beta}^{(1)}}(\kappa, t), \widehat{G_{\beta}^{(2)}}(\kappa, t)$, and then invert these Fourier transforms.

Strategy (S1): Recalling the Fourier transform pair,

$$
\frac{a_{j}}{b+\kappa^{2}} \stackrel{\mathscr{F}}{\leftrightarrow} \frac{a_{j}}{2 b^{1 / 2}} \mathrm{e}^{-\left.|x|\right|^{1 / 2}}, \quad b>0,
$$

and setting $a_{j}=s^{\beta-j}, b=s^{\beta}$ we get

$$
\widetilde{G_{\beta}^{(j)}}(x, s)=\frac{s^{\beta / 2-j}}{2} \mathrm{e}^{-\left.|x|\right|^{\beta / 2}}, \quad j=1,2 .
$$

Strategy (S2): Recalling the Laplace transform pair, see e.g. [8,20],

$$
\frac{s^{\beta-j}}{s^{\beta}+c} \stackrel{\mathscr{L}}{\leftrightarrow} t^{j-1} E_{\beta, j}\left(-c t^{\beta}\right), \quad c>0,
$$

where $E_{\beta, j}$ denotes the two-parameter Mittag-Leffler function ${ }^{2}$ and setting $c=\kappa^{2}$ we get

$$
\widehat{G_{\beta}^{(j)}}(\kappa, t)=t^{j-1} E_{\beta, j}\left(-\kappa^{2} t^{\beta}\right), \quad j=1,2 .
$$

The strategy (S1) has been followed by Mainardi [11-13] to obtain the first Green function as

\footnotetext{
${ }^{2}$ The Mittag-Leffler function $E_{\beta, \mu}$ with $\beta, \mu>0$ is an entire transcendental function of order $\rho=1 / \beta$, defined in the complex plane by the power series

$$
E_{\beta, \mu}(z):=\sum_{n=0}^{\infty} \frac{z^{n}}{\Gamma(\beta n+\mu)}, \quad \beta, \mu>0, \quad z \in \mathbb{C} .
$$

Originally, at the beginning of 1900, Mittag-Leffler introduced and investigated (in five notes from 1902 to 1905 ) the function

$$
E_{\alpha}(z):=\sum_{n=0}^{\infty} \frac{z^{n}}{\Gamma(\alpha n+1)}, \quad \alpha>0, \quad z \in \mathbb{C},
$$

as an instructive example of entire function that generalizes the exponential. For more information on the Mittag-Leffler-type functions the reader may consult the classical handbook of the Bateman Project [3, vol. 3, Chapter 18] and e.g. [7,8,10,14,20,21].
} 


$$
G_{\beta}^{(1)}(x, t)=\frac{1}{2} t^{-\beta / 2} M_{\beta / 2}\left(|x| / t^{\beta / 2}\right), \quad-\infty<x<+\infty, \quad t \geqslant 0,
$$

where $M_{\beta / 2}$ denotes the so-called $M$ function of order $\beta / 2$, see also [9,20], which is a noteworthy case of the Wright function. ${ }^{3}$ As far as the second Green function is concerned, we note from (3.2) that $\widetilde{G_{\beta}^{(2)}}(x, s)=\widetilde{G_{\beta}^{(1)}}(x, s) / s$, so

$$
G_{\beta}^{(2)}(x, t)=\int_{0}^{t} G_{\beta}^{(1)}(x, \tau) \mathrm{d} \tau .
$$

Closed form solutions are found in the special case $\beta=1$ (diffusion equation) and in the limiting case $\beta=2$ (D'Alembert wave equation). We easily recognize for $\beta=1$ :

$$
G_{1}^{(1)}(x, t)=\frac{t^{-1 / 2}}{2 \sqrt{\pi}} \mathrm{e}^{-x^{2} /(4 t)}, \quad G_{1}^{(2)}(x, t)=\frac{t^{1 / 2}}{\sqrt{\pi}} \mathrm{e}^{-x^{2} /(4 t)}-\frac{x}{2} \operatorname{erfc}\left(\frac{x}{2 t^{1 / 2}}\right),
$$

where erfc denotes the complementary error function, and, for $\beta=2$ :

$$
G_{2}^{(1)}(x, t)=\frac{\delta(x+t)+\delta(x-t)}{2}, \quad G_{2}^{(2)}(x, t)=\frac{\theta(x+t)-\theta(x-t)}{2},
$$

where $\theta$ denotes the unit-step Heaviside function.

The strategy (S2) has been followed by Gorenflo, Iskenderov \& Luchko [4] and by Mainardi, Luchko \& Pagnini [15] to obtain the first Green function of the more general space-time-fractional diffusion equations. For the determi-

\footnotetext{
${ }^{3}$ The function $M_{v}(z)$ is defined for any order $v \in(0,1)$ and $\forall z \in \mathbb{C}$ by

$$
M_{v}(z):=\sum_{n=0}^{\infty} \frac{(-z)^{n}}{n ! \Gamma[-v n+(1-v)]}, \quad 0<v<1, \quad z \in \mathbb{C} .
$$
}

It turns out that $M_{v}(z)$ is an entire function of order $\rho=1 /(1-v)$, which provides a generalization of the Gaussian and of the Airy function. In fact we obtain

$$
M_{1 / 2}(z)=\frac{1}{\sqrt{\pi}} \exp \left(-z^{2} / 4\right), \quad M_{1 / 3}(z)=3^{2 / 3} \operatorname{Ai}\left(z / 3^{1 / 3}\right) .
$$

The $M$ function is a special case of the Wright function defined by the series representation, valid in the whole complex plane,

$$
\Phi_{\lambda, \mu}(z):=\sum_{n=0}^{\infty} \frac{z^{n}}{n ! \Gamma(\lambda n+\mu)}, \quad \lambda>-1, \quad \mu \in \mathbb{C}, \quad z \in \mathbb{C} .
$$

Indeed, we recognize

$$
M_{v}(z)=\Phi_{-v, 1-v}(-z), \quad 0<v<1 .
$$

Originally, Wright introduced and investigated this function with the restriction $\lambda \geqslant 0$ in a series of notes starting from 1933 in the framework of the asymptotic theory of partitions. Only later, in 1940, he considered the case $-1<\lambda<0$. We note that in the handbook of the Bateman Project $[3$, vol. 3, Chapter 18], presumably for a misprint, $\lambda$ is restricted to be non-negative. For more information on the Wright-type functions in time-fractional diffusion equations the interested reader may consult e.g. [5,6,9]. 
nation of the reduced Green functions $K_{\beta}^{(j)}(x)=G_{\beta}^{(j)}(x, 1)$ we can restrict our attention to $x>0$, and thus write in view of (3.4) and (2.7)

$$
K_{\beta}^{(j)}(x)=\frac{1}{\pi} \int_{0}^{\infty} \cos (\kappa x) E_{\beta, j}\left(-\kappa^{2}\right) \mathrm{d} \kappa, \quad j=1,2 .
$$

Following the method outlined in [4] and [15] we can invert the Fourier transforms in (3.9) and obtain

$$
K_{\beta}^{(j)}(x)=\frac{1}{2 x} \frac{1}{2 \pi \mathrm{i}} \int_{\gamma-\mathrm{i} \infty}^{\gamma+\mathrm{i} \infty} \frac{\Gamma(1-s)}{\Gamma(j-\beta s / 2)} x^{s} \mathrm{~d} s, \quad 0<\gamma<1, \quad j=1,2 .
$$

The above integral is a particular Mellin-Barnes integral according to a usual terminology. ${ }^{4}$ The readers who are acquainted with the high transcendental Fox $H$ functions can recognize in the R.H.S. of (3.10) the representation of a certain function of this class see e.g. [1,9,10,17-19,21-23]. Unfortunately, as far as we know, computing routines for this general class of special functions are not yet available. Here, following the approach adopted in [15], we intend to compute the (reduced) Green functions in any space domain by matching a convergent power series (suitable for small $|x|$ ) with an asymptotic representation (suitable for large $|x|$ ).

In order to obtain the convergent power series we transform the original contour in (3.10) to the loop $L_{+\infty}$ encircling all the poles $s_{n}=1+n, n \in \mathbb{N}_{0}$ of the function $\Gamma(1-s)$ and apply the residue theorem. We obtain

$$
K_{\beta}^{(j)}(x)=\frac{1}{2} \sum_{n=0}^{\infty} \frac{(-x)^{n}}{n ! \Gamma[-\beta n / 2+(j-\beta / 2)]}, \quad j=1,2 .
$$

The asymptotic representation can be obtained by using the arguments by Braaksma [2] (see also [15]) and turns out to be

$$
K_{\beta}^{(j)}(x) \sim A_{j} x^{a} \mathrm{e}^{-b x^{c}}, \quad x \rightarrow+\infty,
$$

where

$$
\left\{\begin{array}{l}
A_{1}=\left\{2 \pi(2-\beta) 2^{\beta /(2-\beta)} \beta^{(2-2 \beta) /(2-\beta)}\right\}^{-1 / 2}, \\
A_{2}=\left\{2 \pi(2-\beta) 2^{(5 \beta-8) /(2-\beta)} \beta^{(6-4 \beta) /(2-\beta)}\right\}^{-1 / 2},
\end{array}\right.
$$

\footnotetext{
${ }^{4}$ In this respect the interested reader can find in e.g. [3], the discussion on the general conditions of convergence for the typical Mellin-Barnes integral, based on the asymptotic representation of the gamma function. The names refer to the two authors, who in the beginning of the past century developed the theory of these integrals using them for a complete integration of the hypergeometric differential equation. However, as pointed out in [3, vol. 1, Chapter 1, Section 1.19, p. 49], these integrals were first used by the Italian mathematician S. Pincherle in 1888, see [16].
} 


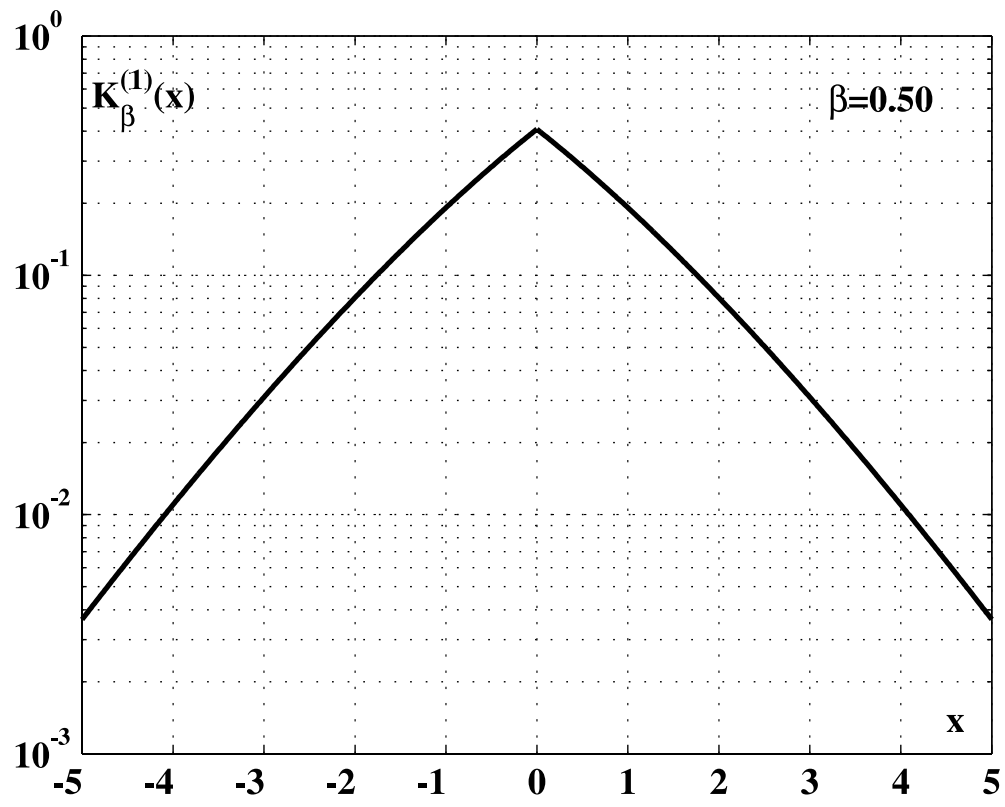

Fig. 1. Plot of $K_{\beta}^{(1)}(x)$ for $\beta=0.5$.

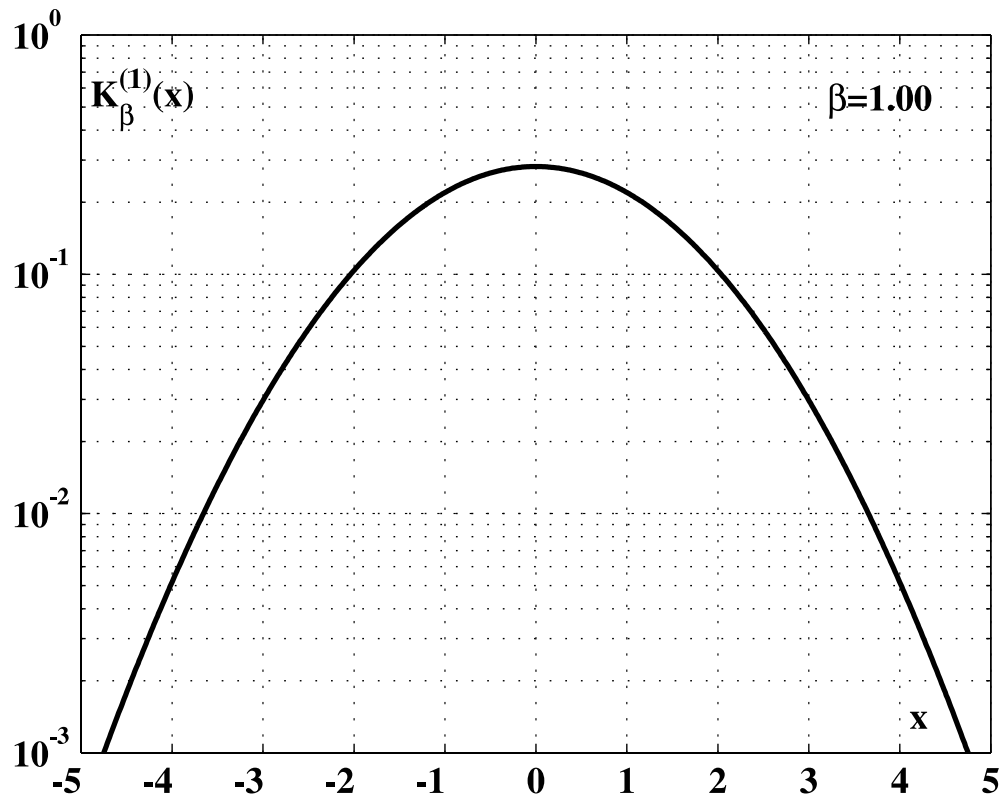

Fig. 2. Plot of $K_{\beta}^{(1)}(x)$ for $\beta=1$. 


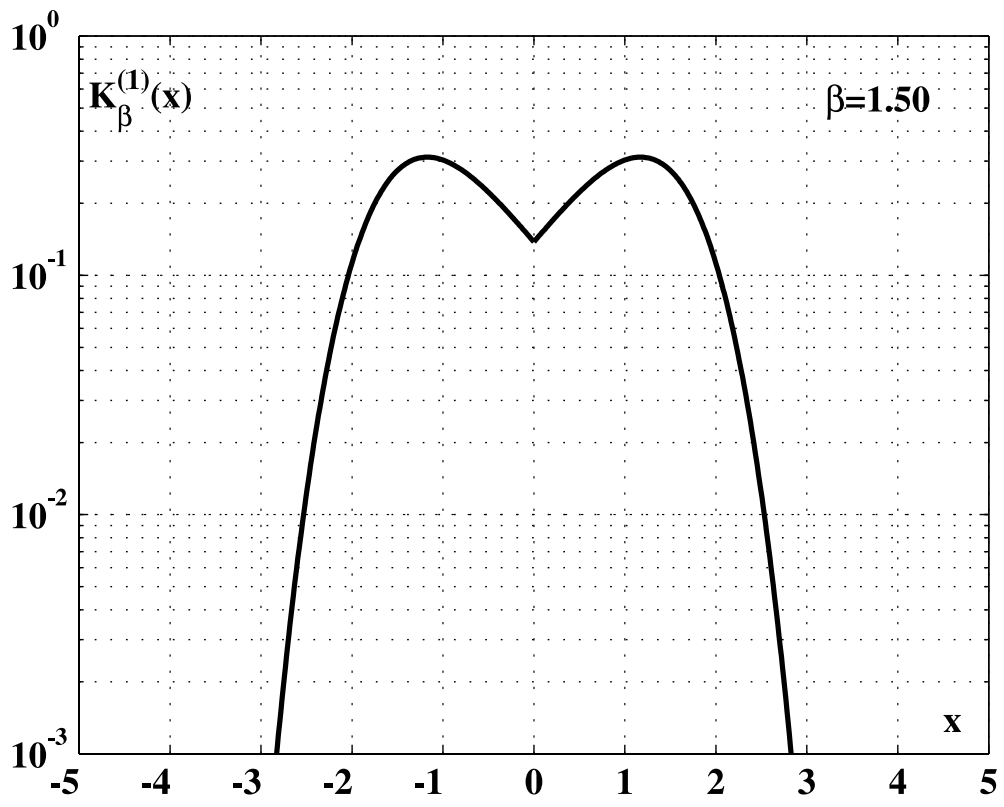

Fig. 3. Plot of $K_{\beta}^{(1)}(x)$ for $\beta=1.5$.

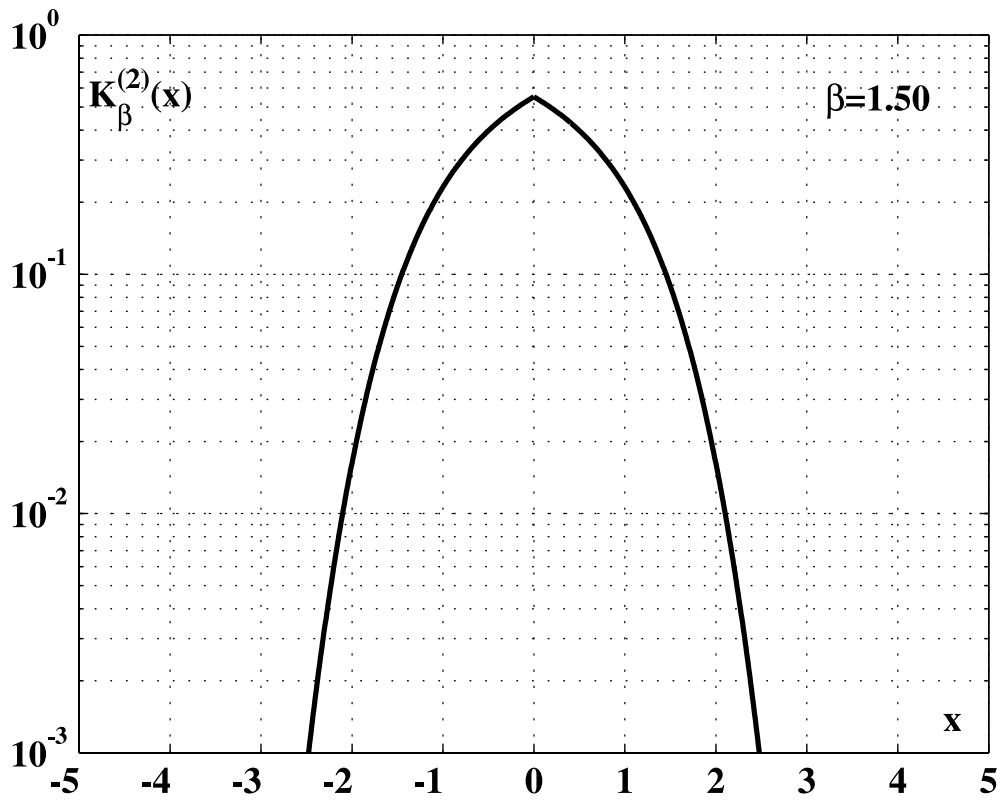

Fig. 4. Plot of $K_{\beta}^{(2)}(x)$ for $\beta=1.5$. 


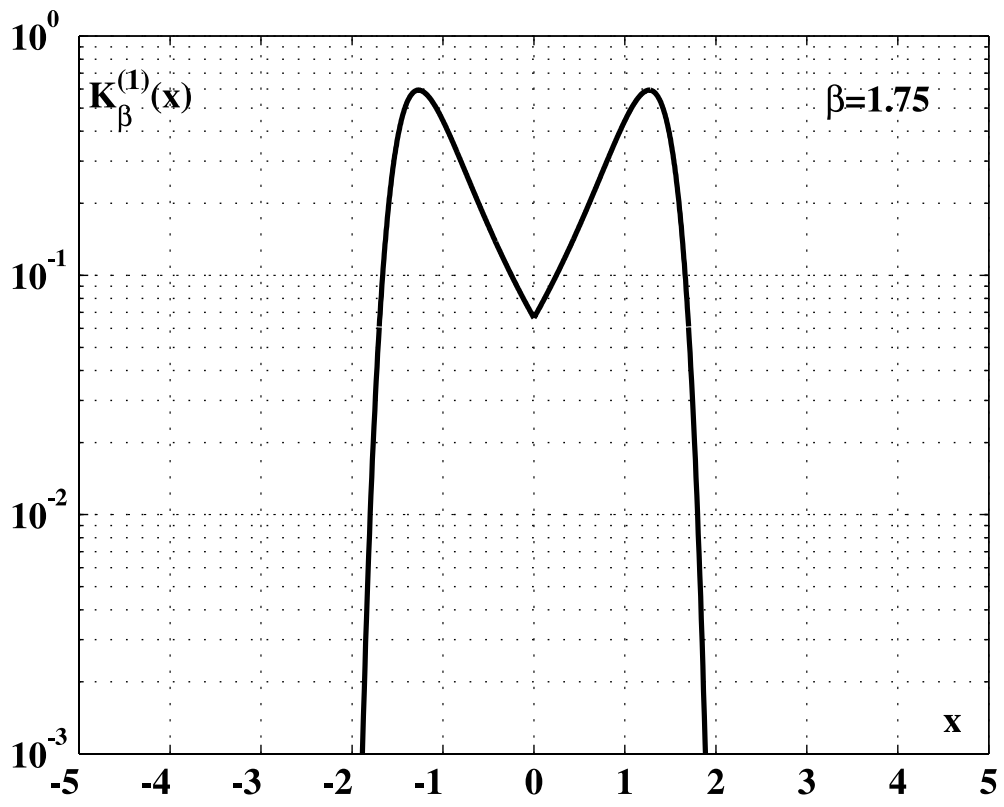

Fig. 5. Plot of $K_{\beta}^{(1)}(x)$ for $\beta=1.75$.

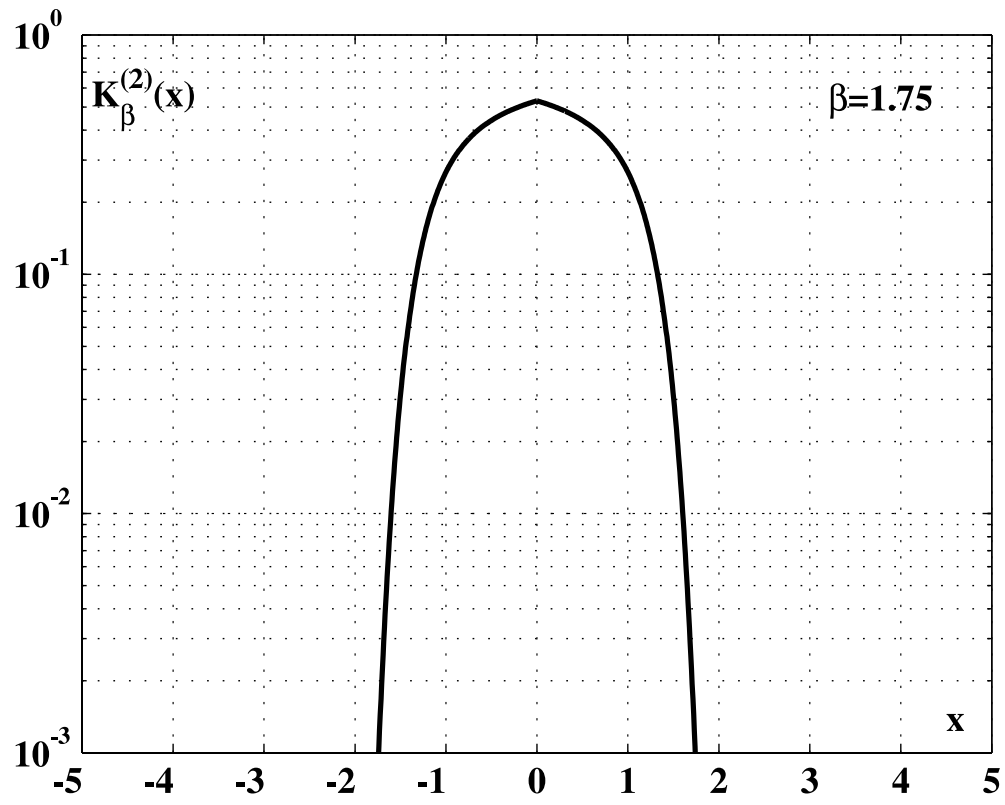

Fig. 6. Plot of $K_{\beta}^{(2)}(x)$ for $\beta=1.75$. 


$$
a=\frac{2 \beta-2}{2(2-\beta)}, \quad b=(2-\beta) 2^{-2 /(2-\beta)} \beta^{\beta /(2-\beta)}, \quad c=\frac{2}{2-\beta} .
$$

We find it convenient to exhibit in Figs. 1-6 a few plots of the reduced Green functions $K_{\beta}^{(j)}(x)$ for some "characteristic" values of the parameter $\beta$. The plots are drawn by using the MATLAB system for the values of the independent variable $x$ in the range $|x| \leqslant 5$. To give the reader a better impression about the behaviour of the tails, the logarithmic scale is adopted. Both the Green functions turn out to be non-negative and normalized, so they are of the greatest interest in view of their interpretation as probability densities.

\section{Acknowledgements}

Research performed under the auspices of the National Group of Mathematical Physics (G.N.F.M.-I.N.D.A.M.) and partially supported by the Italian Ministry of University (M.I.U.R.) through the Research Commission of the University of Bologna. The authors are grateful to Prof. R. Gorenflo for helpful discussions.

\section{References}

[1] V.V. Anh, N.N. Leonenko, Spectral analysis of fractional kinetic equations with random data, Journal of Statistical Physics 104 (5/6) (2001) 1349-1387.

[2] B.L.J. Braaksma, Asymptotic expansions and analytical continuations for a class of Barnesintegrals, Compositio Mathematica 15 (3) (1962) 239-341.

[3] A. Erdélyi, W. Magnus, F. Oberhettinger, F.G. Tricomi, Higher Transcendental Functions, Bateman Project, vols. 1-3, McGraw-Hill, New York, 1953-1955.

[4] R. Gorenflo, A. Iskenderov, Yu. Luchko, Mapping between solutions of fractional diffusionwave equations, Fractional Calculus and Applied Analysis 3 (1) (2000) 75-86.

[5] R. Gorenflo, Yu. Luchko, F. Mainardi, Analytical properties and applications of the Wright function, Fractional Calculus and Applied Analysis 2 (4) (1999) 383-414.

[6] R. Gorenflo, Yu. Luchko, F. Mainardi, Wright functions as scale-invariant solutions of the diffusion-wave equation, Journal of Computational and Applied Mathematics 118 (2000) 175-191.

[7] R. Gorenflo, Yu. Luchko, S. Rogozin, Mittag-Leffler type functions: notes on growth properties and distribution of zeros, Pre-print A-04/97, Fachbereich Mathematik und Informatik, Freie Universität, Berlin, 1997. Available from <http://www.math.fu-berlin.de/ publ/index.html .

[8] R. Gorenflo, F. Mainardi, Fractional calculus: integral and differential equations of fractional order, in: A. Carpinteri, F. Mainardi (Eds.), Fractals and Fractional Calculus in Continuum Mechanics, Springer Verlag, Wien and New York, 1997, pp. 223-276. Reprinted in NEWS 010101. Available from <http://www.fracalmo.org $>$.

[9] R. Gorenflo, F. Mainardi, H.M. Srivastava, Special functions in fractional relaxationoscillation and fractional diffusion-wave phenomena, in: D. Bainov (Ed.), Proceedings VIII International Colloquium on Differential Equations, Plovdiv 1997, VSP, Utrecht, 1998, pp. 195-202. 
[10] V. Kiryakova, Generalized Fractional Calculus and Applications, Pitman Research Notes in Mathematics, vol. 301, Longman, Harlow, 1994.

[11] F. Mainardi, The fundamental solutions for the fractional diffusion-wave equation, Applied Mathematics Letters 9 (6) (1996) 23-28.

[12] F. Mainardi, Fractional relaxation-oscillation and fractional diffusion-wave phenomena, Chaos, Solitons and Fractals 7 (1996) 1461-1477.

[13] F. Mainardi, Fractional calculus: some basic problems in continuum and statistical mechanics, in: A. Carpinteri, F. Mainardi (Eds.), Fractals and Fractional Calculus in Continuum Mechanics, Springer Verlag, Wien and New York, 1997, pp. 291-348. Reprinted in NEWS 011201. Available from <http://www.fracalmo.org $>$.

[14] F. Mainardi, R. Gorenflo, On Mittag-Leffler-type functions in fractional evolution processes, Journal of Computational and Applied Mathematics 118 (2000) 283-299.

[15] F. Mainardi, Yu. Luchko, G. Pagnini, The fundamental solution of the space-time fractional diffusion equation, Fractional Calculus and Applied Analysis 4 (2) (2001) 153-192, Reprinted with permission in NEWS 010401. Available from <http://www.fracalmo.org>.

[16] F. Mainardi, G. Pagnini, Salvatore Pincherle: the pioneer of the Mellin-Barnes integrals, J. Comput. Appl. Math., in press. 6th International Symposium on Orthogonal Polynomials, Special Functions and Applications, Roma-Ostia, Italy, June 18-22, 2001.

[17] O.I. Marichev, Handbook of Integral Transforms of Higher Transcendental Functions, Theory and Algorithmic Tables, Chichester, Ellis Horwood, 1983.

[18] A.M. Mathai, R.K. Saxena, The $H$-function with Applications in Statistics and Other Disciplines, Wiley Eastern Ltd., New Delhi, 1978.

[19] R. Metzler, J. Klafter, The random walk's guide to anomalous diffusion: a fractional dynamics approach, Physics Reports 339 (2000) 1-77.

[20] I. Podlubny, Fractional Differential Equations, Academic Press, New York, 1999.

[21] S.G. Samko, A.A. Kilbas, O.I. Marichev, Fractional Integrals and Derivatives: Theory and Applications, Gordon and Breach Science Publishers, New York and London, 1993 (Translation from the Russian edition, Nauka i Tekhnika, Minsk, 1987).

[22] H.M. Srivastava, K.C. Gupta, S.P. Goyal, The H-Functions of One and Two Variables with Applications, South Asian Publishers, New Delhi and Madras, 1982.

[23] H.M. Srivastava, B.R.K. Kashyap, Special Functions in Queuing Theory and Related Stochastic Processes, Academic Press, New York, 1982. 